\title{
Development of precipitation nowcasting method using geostationary satellite data
}

\author{
A. I. Andreev, N. I. Pererva, M. O. Kuchma \\ Far Eastern Center SRC Planeta, Khabarovsk, 680000, Russia \\ E-mail: a.andreev@dvrcpod.ru
}

\begin{abstract}
The paper considers the development of a model for precipitation field nowcasting using the data obtained from the Himawari- 8 satellite and a GFS numerical forecast model. The nowcasting method employs a convolutional and recurrent neural network architecture. A peculiarity of the developed model is a possibility to make a forecast using no ground-based meteorological radars data. The authors present preliminary research results as exemplified by the precipitation field nowcasting for a 30-minute period and the 60-minute forecast of the cloud cover optical depth distribution. Finally, the paper outlines the areas for further research with the account to the identified drawbacks of the existing forecasting algorithm software implementation.
\end{abstract}

Accepted: 15.09 .2020

DOI: 10.21046/2070-7401-2020-17-6-18-22

\begin{abstract}
1. Introduction
Weather nowcasting with a high spatiotemporal resolution is currently one of the most actively studied areas in hydrometeorology. Timely and precise forecasts of dangerous weather events are crucial for the operation of urban services, regional aviation, agriculture, as well as issuing community warnings.

The increased interest to nowcasting from the scientific community is facilitated by the continuously improving quality and growing amount of data obtained due to the commissioning of new satellites and ground meteorological radars as well as the development of computational forecasting methods. At the same time the major part of the current research largely uses radar data for nowcasting as being the most plausible [1-4] in comparison with the data generated by the computational forecast models and geostationary satellites. Despite the fact that over the last decades the model forecasts precision has considerably increased, it requires a lot of computational and time resources to conduct computations with a high spatiotemporal resolution comparable with the radar data resolution.

In view of the insufficient coverage of the Far East territory with the data of ground meteorological radars satellite data become increasingly important. Thus, this paper considers Himawari- 8 with an on-board AHI (Advanced Himawari Imager) device having a high spatial resolution (up to $2 \mathrm{~km}$ ) and the shooting interval of $10 \mathrm{~min}$ (it allows partially compensating for the drawbacks of ground observation). In addition, an effective range of radar action is significantly limited while geostationary satellites can be used to obtain a global scale image.

The present paper dwells upon the development of a nowcasting model for the cloud cover and precipitation dynamics. The key peculiarity of the developed model is a possibility of precipitation nowcasting using no radar data. The paper comprises the preliminary research results and outlines further prospects of research in the area.
\end{abstract}




\section{Nowcasting methods}

Recently, a lot of nowcasting models have been presented, and a large part of them can be provisionally divided into two big groups: the methods based upon the algorithms of optical flow and the models based upon machine learning (ML).

Optical flow method varieties are successfully applied for the nowcasting of cloudiness drifting and precipitation fields for a short period (up to 3 hours) [5,6]. These methods are based upon the assumption that the values of each specific pixel, pixel group or their gradient are not subject to significant changes in time while their displacement on a pair of images is negligible. The optical flow algorithm operation results in a map of pixel motion vectors for a pair of images. This method is relatively simple in terms of implementation, has a high operation speed, is suitable for shot interpolation and extrapolation and demonstrates high precision when large-scale cloudiness is forecast [6].

The drawbacks of optical flow algorithms can be logically derived from the aforementioned assumptions about the limited observed object changeability. Convective cloudiness is a complex dynamic system and can significantly change within a short time passing through the stages of growth and disintegration which contradicts with these assumptions. It entails a rapid growth in the number of errors at the nowcasting of such cloud drifting [5].

Another approach to nowcasting consists in the use of machine learning (ML) algorithms. The advantage of this approach is its flexibility which gives a possibility to use complex structures as input data, for example, a three-dimensional distribution of temperature, humidity, reflecting property values by radar data, etc. [8]. Also, ML algorithms allow modeling non-linear interconnections between the properties which is their advantage of the optical flow algorithms.

Convolution neural networks (CNN) and recurrent neural networks (RNN) have become the most wide-spread for ML nowcasting, with CNN suitable for image processing and RNN having the memory effect. The effort of combining the advantages of CNN and RNN resulted in the development of hybrid architectures such as ConvLSTM, PredNet, etc., making them the most suitable for nowcasting $[4,9,10]$.

Today a neural network approach is being successfully applied by the combined data from radars, satellites and computational forecast models [10]. However, such systems are efficient only for the territory where such system has been previously trained and, in most cases, where ground radar data are available [2]. The present paper describes the process of developing a precipitation nowcasting model for the Far East region of Russia providing no radar data are available.

\section{Input data}

To train a neural network model, the authors formed a set of data including the AHI channels of a geostationary satellite Himawari-8 in the visible and IR ranges $(0.6,2.3,3.9,6.2,9.6,12.4$ and $13.3 \mathrm{um}$ ), forecast fields of a GFS (Global Forecasting System) numerical model, such as air temperature, dew point and a relative humidity on the standard isobar levels as well as the terrain above-sea level. All the data used were obtained for the territory of the Far East region covering the periods of July 2018 and 2019 during the daytime.

The set of training data consists of 133 thousand shot sequences, each of them including 13 images $128 \times 128$ pixels with a horizontal resolution $2 \mathrm{~km}$ and a time interval of 10 minutes. As the GFS model has a three-hour forecast interval (step), the model data are interpolated over time up to 10 minutes with the use of the optical flow algorithm by a modified Brox's method [7].

The algorithm output data are binarized maps of precipitation fields for up to 1 hour duration. The map data are formed by the precipitation detection algorithm described in the next section of the paper.

\section{The method for cloud and precipitation forecasting}

For nowcasting of precipitation fields and cloudiness drift the authors use the method based on the convolution-recurrent neural network PredNet [11]. This architecture has been developed for the extrapolation of the video sequence images and further successfully adapted for nowcasting tasks on the data of ground meteorological radars [10].

The key components of the architecture PredNet are ConvLSTM cells (Convolutional Long ShortTerm Memory) capable of retaining the information received at the previous levels of data sequence 
[11]. A characteristic feature of ConvLSTM cells against "classical" LSTM cells is a more efficient image processing as there are convolution layers at the cell input [4].

A sequence in the form of the 4-dimensional tensor Xt,w,h,p is sent to the input of the abovementioned neural network, where $t$ - image number $w, h$ - image height and width, $p-$ parameter (isobar level temperature, IR channel, etc.). The output data are the images $t+n$ where $n-$ number of the forecast image. This paper considers a nowcasting method where each number $\mathrm{n}$ corresponds to a specific neural network model trained for some fixed forecast period. Within further research the authors will also consider the options when the only neural network model is used for a forecast of any duration.

To compensate for the absence of micro-wave range data, the research studies a possibility of developing such an algorithm to estimate precipitation fields and their instantaneous intensity with the use of IR range data as well as the information obtained by means of numerical forecast models. At the current research stage, the authors apply the algorithm based upon an empirical dependence of a potential precipitation cloudiness and its height on precipitation intensity as a precipitation detector [12]. The obtained intensity value is rather approximated, and the algorithm for its obtaining should be specified.

\section{Preliminary research results}

The papers considers the possibility of precipitation field and cloud drifting nowcasting, and thus, two neural network models are trained. The first model uses a full set of input parameters enumerated in the section "reference data" and is intended for precipitation nowcasting. The second model is used in the optical flow "mode": cloud drifting forecast is made only on the basis of one visible channel or the cloudiness parameter.

Figure 1 shows the results of the nowcasting algorithm operation in the optical flow "mode" for the period of 60 minutes; the values of cloudiness optical depth are used as a forecast parameter. In general, the authors point to a right direction of cloud drifting; however, the range of problems can be also identified.
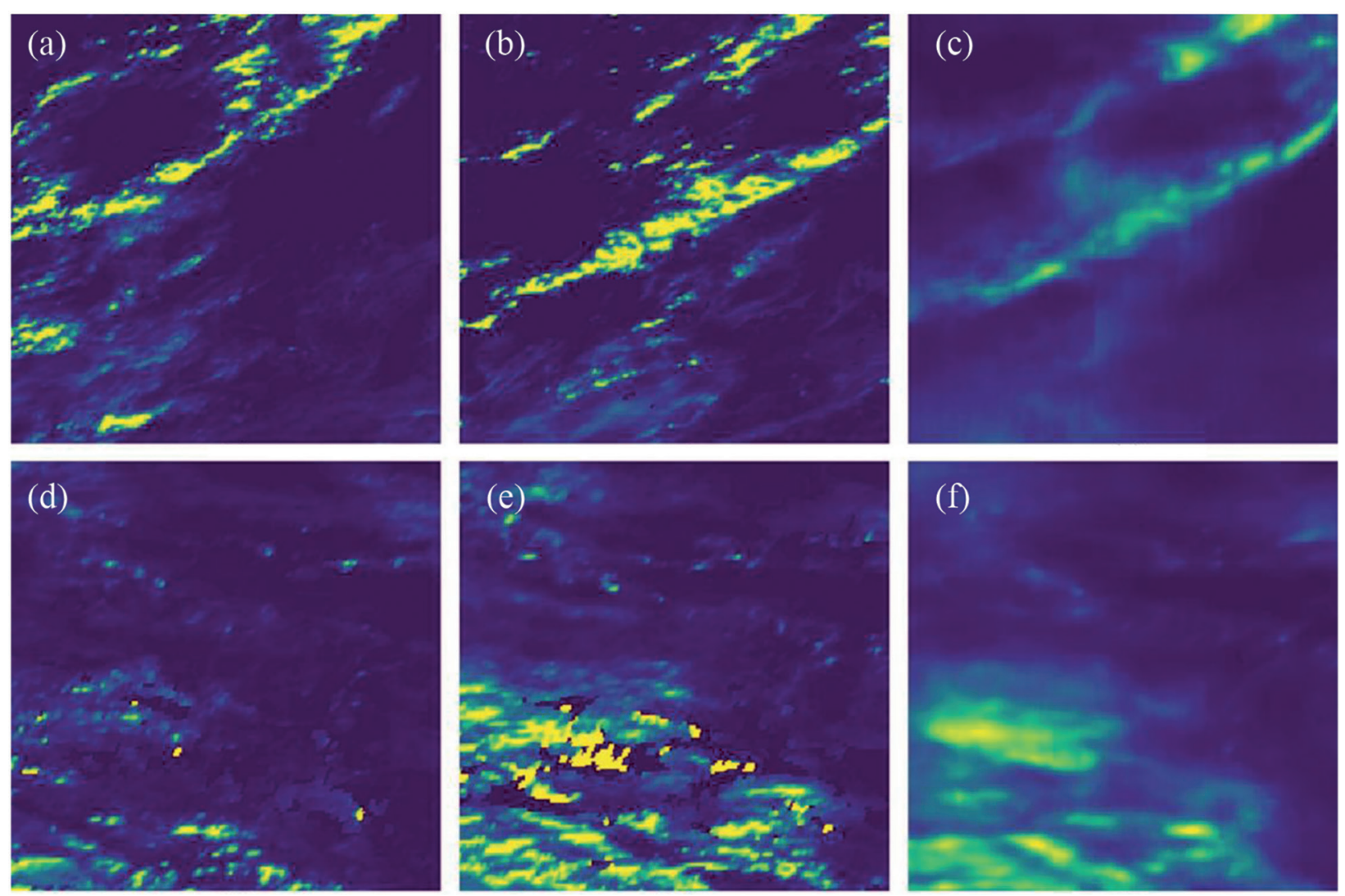

Figure 1. Optical depth forecasting: (a), (d) - opitcal depth distribution at the initial moment; (b), (e) - real optical depth distribution in 60 minutes; (c), (f) - forecasting results in 60 minutes. 
The upper row in figure 1 demonstrates the clouds motion left-to-right. Here it is evident that the cloudiness occurring in the left part of figure $1 \mathrm{~b}$ is absent in the forecast image (Figure 1c). This problem is caused by the fact that at the current development stage the algorithm does not take account for the information beyond the forecast region. Another problem is the insufficiently precise forecast of optical depth values which manifests in the pixel intensity and some image "blurriness". Both problems are the drawbacks of the current implementation of the nowcasting model, and the researchers keep working to eliminate them.

The results of precipitation field distribution nowcasting for 30 minutes in comparison with the actual algorithm data for their detection are provided in figure 2. When comparing the results in the general case one can point that most forecast areas coincide with the actual precipitation distribution by the algorithm data for their detection. However, the nowcasting algorithm is prone to overestimation of the size of precipitation forming cloudiness regions. A more detailed forecasting is one of the further research areas. The resolution of this problem is defined not only by the precision of the operation of the nowcasting model itself but also by the quality of the detection algorithm operation, the data of which are used for neural network training.
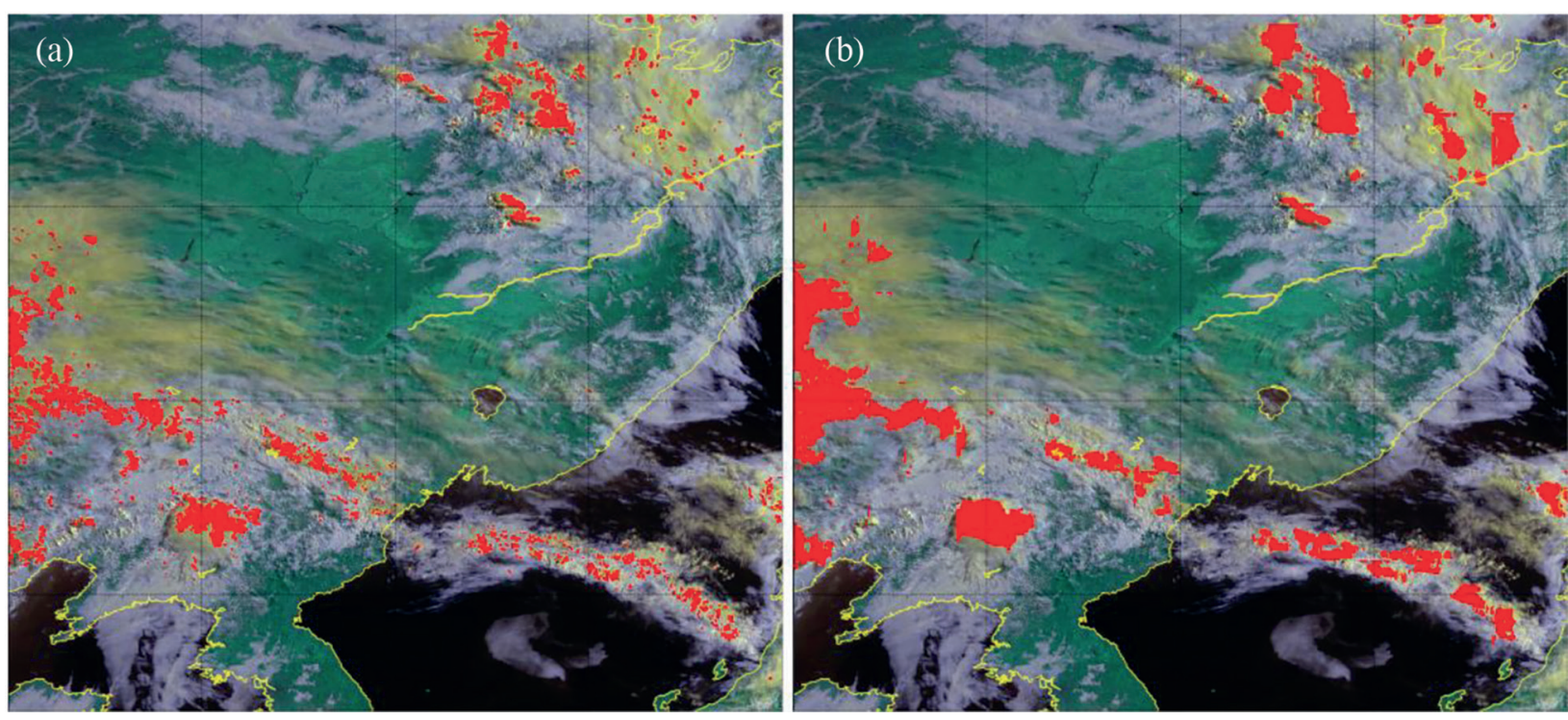

Figure 2. Precipitation fields nowcasting in 30 minutes starting from 22:50 UTC 15 July 2018: (a) - real precipitation fields distribution; (b) - nowcasting results.

\section{Conclusion}

The paper provides the preliminary research results on precipitation and cloud drifting nowcasting based on the data obtained from the Himawari- 8 geostationary satellite and a computational forecasting GFS model. The nowcasting algorithm is based on the model of a convolution-recurrent neural network PredNet previously developed for optical flow tasks.

The specified drawbacks of the current algorithm implementation point to the necessity of further research conduct. One of the promising areas for model improvement is the improvement of the PredNet architecture by means of LSTM blocks' modification to increase the duration of forecasts and their precision. In addition, neural network training should be conducted by means of the generative-adversarial network method (Conditional Generative-Adversarial Networks) [13].

\section{Acknowledgements}

In the process of the nowcasting model development the data of CCU "FEB RAS Data Center" (Sorokin et al., 2017). The computations were conducted by the methods and technologies developed with the funding of the Russian Foundation for Basic Research (RFBR) in the framework of a scientific project No. 18-29-03196. 


\section{References}

[1] Agrawal S., Barrington L., Bromberg C., Burge J., Gazen C., Hickey J., Machine Learning for Precipitation Nowcasting from Radar Images, arXiv preprint arXiv:1912.12132, 2019, 6 p., available at: https://arxiv.org/pdf/1912.12132.pdf.

[2] Lebedev V., Ivashkin V., Rudenko I., Ganshin A., Molchanov A., Ovcharenko S., Grokhovetskiy R., Bushmarinov I., Solomentsev D., Precipitation nowcasting with satellite imagery, 25th ACM SIGKDD Intern. Conf. Knowledge Discovery and Data Mining, Proc., 2019, pp. 2680-2688, available at: https:// dl.acm.org/doi/10.1145/3292500.3330762.

[3] Woo W., Wong W., Operational Application of Optical Flow Techniques to Radar-Based Rainfall Nowcasting, Atmosphere, 2017, Vol. 8(3), 48, 20 p., DOI: 10.3390/atmos8030048.

[4] Xingjian S. H. I., Chen Z., Wang H., Yeung D. Y., Wong W. K., Woo W. C., Convolutional LSTM Network: A Machine Learning Approach for Precipitation Nowcasting, Advances in Neural Information Processing Systems, 2015, Vol. 28, pp. 802-810, available at: https://papers.nips.cc/paper/2015/file/07563a3fe3bbe7 e3ba84431ad9d055af-Paper.pdf.

[5] Sirch T., Bugliaro L., Zinner T., Möhrlein M., Vazquez-Navarro M., Cloud and DNI nowcasting with MSG/SEVIRI for the optimized operation of concentrating solar power plants, Atmospheric Measurement Techniques, 2017, Vol. 10(2), pp. 409-429, available at: https://amt.copernicus.org/articles/10/409/2017/ amt-10-409-2017.pdf.

[6] Liu Y., Xi D.G., Li Z. L., Hong Y., A new methodology for pixel-quantitative precipitation nowcasting using a pyramid Lucas Kanade optical flow approach, J. Hydrology, 2015, Vol. 529, pp. 354-364.

[7] Simonenko E. V., Chudin A. O., Davidenko A. N., The differential method for calculation of cloud motion vectors, Russian Meteorology and Hydrology, 2017, Vol. 42(3), pp. 159-167.

[8] Zhang W., Han L., Sun J., Guo H., Dai J., Application of multi-channel 3D-cube successive convolution network for convective storm nowcasting, IEEE Intern. Conf. Big Data (BigData), 2019, pp. 1705-1710.

[9] Akbari Asanjan A., Yang T., Hsu K., Sorooshian S., Lin J., Peng Q., Short-Term Precipitation Forecast Based on the PERSIANN System and LSTM Recurrent Neural Networks, J. Geophysical Research: Atmospheres, 2018, Vol. 123(22), pp. 12543-12563.

[10] Sato R., Kashima H., Yamamoto T., Short-term precipitation prediction with skip-connected PredNet, Intern. Conf. Artificial Neural Networks, Proc., 2018, pp. 373-382.

[11] Lotter W., Kreiman G., Cox D., Deep Predictive Coding Networks for Video Prediction and Unsupervised Learning, arXiv print arXiv:1605.08104, 2016, 18 p., available at: https://arxiv.org/pdf/1605.08104.pdf.

[12] Alekseeva A.A., Bukharov M.V., Diagnosis of Precipitation and Thunderstorms from Measurements of Outgoing Heat Radiation of a Cloud Cover from Geostationary Satellites, Russian Meteorology and Hydrology, 2005, Vol. 6, pp. 20-26.

[13] Mirza M., Osindero S., Conditional Generative Adversarial Nets, arXiv preprint arXiv:1411.1784, 2014, 7 p., available at: https://arxiv.org/pdf/1411.1784.pdf.

[14] Sorokin A.A., Makogonov S. I., Korolev S.P., The Information Infrastructure for Collective Scientific Work in the Far East of Russia, Scientific and Technical Information Proc., 2017, Vol. 4, pp. 302-304. 